\title{
Time-Dependent Dissipative Massive Scalar Field
}

\author{
Marjan Jafari \\ Department of Physics, Imam Khomeini International University, Qazvin, Iran. \\ Corresponding author email: $\underline{\text { M.Jafari@sci.ikiu.ac.ir }}$
}

Regular paper: Received: Jan. 3, 2021, Revised: Aug. 8, 2021, Accepted: Aug. 21, 2021, Available Online: Aug. 23, 2021, DOI: 10.52547/ijop.15.1.49

\begin{abstract}
In this work, we investigate a timedependent dissipative scalar field. Inspired by the Bateman lagrangian, we showed a procedure for quantizatizing the massive scalar field with the time-dependent dissipative term. So, the properties of a quantum dissipative scalar field are analyzed by the Caldirola-Kanai model. Also, we construct the Hilbert space for the system and calculate the wave eigen-functions and probability distribution of the system.
\end{abstract}

KEYWORDS: Bateman Lagrangian, CaldirolaKanai model, Dissipative system, Massive scalar field.

\section{INTRODUCTION}

In the last decades many techniques have been developed to describe the dissipative system in to the framework of quantum mechanics. Many interesting physical phenomena arise when quantum systems are subjected to the influence of external time-dependent conditions. Timedependent systems appear in several applications in physics such as ion traps [1], [2], [3], optical cavities [4], and to perform algorithms in quantum computation [5], [6].

A scalar field is a subject that playing an important role in classical and quantum mechanics. The scalar fields appear in various branches of physics. A dissipative scalar field with time-dependent damping factors can be used in describing time-dependent dissipative systems. Most of the physical systems are damping systems [7], [8].

There are many approaches to take into account dissipation in quantum theory, for example the phenomenological [9] and canonical approach
[10]. In these approaches, the interaction between the system and its environment is defined such that irreversible energy flows from the system to its environment results.

The main obstacle in quantizing a dissipative field is that a Lagrangian or a Hamiltonian is required generate the system's quantum evolution and the quantum evolution of the system. This leads to difficulties in implementing the canonical commutation relations if we cannot include a heath bath [11], [12]. Various approaches to quantize a dissipative system have been introduced.

In this work, we showed a procedure for quantizing the massive scalar field with timedependent dissipative terms. In the canonical approach, which we follow here, the whole system is described by a total Lagrangian. Inspired by the Bateman Lagrangian [13], a time-dependent Lagrangian is proposed for a dissipative massive scalar field.

We also construct the Hilbert space of the system and calculate the wave eigen-functions. The wave functions $\psi \mathrm{n}$ are time-dependent and lead, in the general case, to time-dependent expectation values and uncertainty relations for the canonical operators. The properties of a quantum dissipative scalar field are analyzed by the Caldirola-Kanai model [14]. The timedependent creation and annihilation operators are proposed.

\section{Massive Scalar Field}

In the present article, inspired by the Bateman lagrangian, we present a time-dependent 
lagrangian for a dissipative massive scalar field as follows

$$
L=\frac{1}{2}\left(\partial_{t} \varphi^{2}(x, t)-m^{2} \varphi^{2}(x, t)\right) e^{\gamma(t)},
$$

From Euler-Lagrange equations, we find the classical equation of motion for the field as

$$
\partial_{t}^{2} \varphi(x, t)+\dot{\gamma}(t) \varphi(x, t)+m^{2} \varphi(x, t)=0,
$$

Which describes the dissipative massive scalar field. Using the transformation

$$
\psi(x, t)=\varphi(x, t) e^{\gamma(t) / 2},
$$

The Lagrangian is then transformed as

$$
L=\frac{1}{2}\left(\partial_{t} \psi(x, t)-\dot{\gamma}(t) \psi(x, t)\right)^{2}-\frac{m^{2}}{2} \psi^{2}(x, t)
$$

In this step, we want to quantize the model via a canonical quantization approach and find similar coupled differential equations for the quantum fields which we are interested in.

From the Lagrangian density, the conjugate momentum corresponding to the field is defined by

$$
\hat{\pi}_{\psi}(x, t)=-\frac{\partial L}{\partial\left(\partial_{t} \hat{\psi}\right)}=\partial_{t} \hat{\psi}(x, t)-\dot{\gamma}(t) \hat{\psi}(x, t)
$$

The equal-time quantization rule is

$$
\left[\hat{\psi}(x, t), \hat{\pi}\left(x^{\prime}, t\right)\right]=i \hbar \delta\left(x-x^{\prime}\right),
$$

Having the Lagrangian and conjugate momentum, we can find the corresponding Hamiltonian as

$$
\begin{aligned}
\hat{H}= & \frac{1}{2} \hat{\pi}_{\psi}^{2}(x, t)+\frac{1}{2}\left(m^{2}-\frac{\dot{\gamma}^{2}(t)}{4}\right) \hat{\psi}^{2}(x, t), \\
& +\frac{1}{2} \dot{\gamma}(t) \hat{\psi}(x, t) \hat{\pi}_{\psi}(x, t)
\end{aligned}
$$

Where

$$
\Omega=\sqrt{m^{2}-\frac{\dot{\gamma}^{2}(t)}{4}} .
$$

In Heisenberg picture, one finds the equations of motion as operator analogs of classical equations of motion

$$
\partial_{t} \hat{\psi}(x, t)=\frac{i}{\hbar}[H, \hat{\psi}]=\hat{\pi}_{\psi}(x, t)+\frac{1}{2} \dot{\gamma}(t) \hat{\psi}(x, t),
$$

and

$$
\begin{aligned}
& \partial_{t} \hat{\pi}_{\psi}(x, t)=\frac{i}{\hbar}\left[H, \hat{\pi}_{\psi}\right]=, \\
& -\Omega^{2} \hat{\psi}(x, t)-\frac{1}{2} \dot{\gamma}(t) \hat{\pi}_{\psi}(x, t),
\end{aligned}
$$

By substituting Eq. (9) in (8), obtained

$\partial_{t}^{2} \hat{\psi}(x, t)+\left(m^{2}-\frac{1}{2} \ddot{\gamma}(t)\right) \hat{\psi}(x, t)=0$,

that

$$
\varpi=\sqrt{m^{2}-\frac{1}{2} \ddot{\gamma}(t)}
$$

Now we can introduce the pairs of the creation and annihilation operators as follows

$$
\begin{aligned}
& \hat{b}=\sqrt{\frac{\varpi}{2 \hbar}}\left(\hat{\psi}(x, t)+\frac{i}{\varpi} \hat{\pi}(x, t)\right), \\
& \hat{b}^{\dagger}=\sqrt{\frac{\varpi}{2 \hbar}}\left(\hat{\psi}(x, t)-\frac{i}{\varpi} \hat{\pi}(x, t)\right),
\end{aligned}
$$

The time-dependent creation and annihilation operators are proposed here and it can be shown that the creation and annihilation operators satisfy the usual commutation relations and the Hamiltonian can be written in terms of these operators.

The problem is then reduced to the standard quantum free massive scalar field and the frequency of energy Eigen-value is given by $\varpi$

$$
\hat{H}=\hbar \varpi\left(\hat{b}^{\dagger} \hat{b}+\frac{1}{2}\right)
$$




\section{III.EXAMPLES}

We consider a particular case that the frictional coefficient of the system decreases rationally.

$\gamma(t)=\frac{\gamma_{0}}{1+q t}, q>0, \gamma_{0}>0$,

The frequency of this frictional coefficient is

$\varpi=\sqrt{m^{2}-\frac{q^{2} \gamma_{0}}{(1+q t)^{3}}}$,

Another example is the case that the frictional coefficient of the system increase linearly

$\gamma(t)=\gamma_{0} t$

The equation of the motion is converted to this form

$$
\ddot{\psi}(x, t)+\left(m^{2}-\frac{\gamma_{0}^{2}}{4}\right) \psi(x, t)=0,
$$

So, the canonical momentum of the system is

$$
\hat{\pi}_{\psi}(x, t)=\dot{\hat{\psi}}(x, t),
$$

And the Hamiltonian of the system became

$$
\begin{aligned}
\hat{H}= & \frac{1}{2} \hat{\pi}_{\psi}^{2}(x, t)+\frac{1}{2} m^{2} \hat{\psi}^{2}(x, t) \\
& +\frac{1}{2} \gamma_{0} \hat{\psi}(x, t) \hat{\pi}_{\psi}(x, t)
\end{aligned}
$$

The annihilation and creation operators are

$$
\begin{aligned}
& \hat{a}=\sqrt{\frac{\omega_{0}}{2 \hbar}}\left(\hat{\psi}(x, t)+\frac{i}{\omega_{0}} \hat{\pi}_{\psi}(x, t)\right), \\
& \hat{a}^{\dagger}=\sqrt{\frac{\omega_{0}}{2 \hbar}}\left(\hat{\psi}(x, t)-\frac{i}{\omega_{0}} \hat{\pi}_{\psi}(x, t)\right),
\end{aligned}
$$

And the Hamiltonian of the system in terms of the ladder operators is obtained

$$
\hat{H}=\hbar \omega_{0}\left(\hat{a}^{\dagger} \hat{a}+\frac{1}{2}\right)
$$

To obtain the wave function of the field in a position basis, we have

$$
\left\langle x|\hat{\psi}(x, t)| \psi_{0}\right\rangle=\psi_{0}(x),
$$

The ground- state wave function is given by

$\psi_{0}(x)=\left(\frac{\omega_{0}}{\pi \hbar}\right)^{\frac{1}{4}} e^{-\frac{\omega_{0}}{2 \hbar} x^{2}}$,

For any state $\mathrm{n}$, the wave function are expressed in terms of the Hermite polynomial as follows

$$
\begin{aligned}
& \left\langle x|\hat{\psi}(x, t)| \psi_{n}\right\rangle=\psi_{n}(x) \\
& =\frac{(-1)^{n}}{\sqrt{n !}}\left(\frac{\omega_{0}}{\pi \hbar}\right)^{1 / 4} e^{-\frac{\omega_{0}}{2 \hbar} x^{2}} H\left(\sqrt{\frac{\omega_{0}}{\hbar}} x\right)
\end{aligned}
$$

The loss energy wave function of the scalar field is obtained

$$
\begin{aligned}
& \left\langle x|\hat{\varphi}(x, t)| \psi_{n}\right\rangle=\varphi_{n}(x) \\
& =\frac{(-1)^{n}}{\sqrt{n !}}\left(\frac{\omega_{0}}{\pi \hbar}\right)^{1 / 4} e^{-\frac{\omega_{0}}{2 \hbar} x^{2} e^{\gamma_{0} t}} H\left(\sqrt{\frac{\omega_{0}}{\hbar}} x e^{\gamma_{0} t}\right) e^{-i \omega_{0} n t}
\end{aligned}
$$

In this paper we have obtained the exact eigenstates of a dissipative scalar field.

\section{IV.THE SEMI-CALdIROLA- KHANAI HAMILTONIAN}

In this section, inspired by the Caldirola-Khanai Hamiltonian, we introduced a Hamiltonian for the scalar field. Next, by introducing a new canonical transformation, we quantized the dissipative scalar field. Define the canonical transformation

$$
\begin{aligned}
& \hat{\psi}(x, t)=\hat{\xi}(x, t) e^{-\gamma t / 2} \\
& \hat{\pi}_{\psi}(x, t)=\hat{\pi}_{\xi}(x, t) e^{\gamma t / 2},
\end{aligned}
$$

The Hamiltonian equation for the scalar field, using the above canonical transformation, became

$\hat{H}_{c k}=\frac{1}{2} \hat{\pi}_{\xi}^{2}(x, t) e^{\gamma t}+\frac{1}{2} \Omega^{2} \hat{\xi}^{2}(x, t) e^{-\gamma t}$, 
The following equal-time commutation relation is imposed on the field and their conjugate momentum

$\left[\hat{\psi}(x, t), \hat{\pi}_{\psi}\left(x^{\prime}, t\right)\right]=$

$\left[\hat{\xi}(x, t), \hat{\pi}_{\xi}\left(x^{\prime}, t\right)\right]=i \hbar \delta\left(x-x^{\prime}\right)$,

The creation and annihilation operators for these Hamiltonian are defined as follows

$$
\begin{aligned}
& \hat{A}=\sqrt{\frac{\Omega}{2 \hbar}}\left(\hat{\xi}(x, t) e^{-\gamma t / 2}+i \frac{\hat{\pi}_{\xi}(x, t)}{\Omega} e^{\gamma t / 2}\right), \\
& \hat{A}^{\dagger}=\sqrt{\frac{\Omega}{2 \hbar}}\left(\hat{\xi}(x, t) e^{-\gamma t / 2}-i \frac{\hat{\pi}_{\xi}(x, t)}{\Omega} e^{\gamma t / 2}\right),
\end{aligned}
$$

where

$$
\left[\hat{A}, \hat{A}^{\dagger}\right]=1
$$

So the Hamiltonian becomes

$$
\hat{H}_{c k}=\hbar \Omega\left(\hat{A}^{\dagger} \hat{A}+\frac{1}{2}\right)
$$

The Hamiltonian of the dissipative massive scalar field is reduced to the diagonal Hamiltonian with time-dependent creation and annihilation operators.

The coherent states of the system are as follows

$$
\hat{A}\left|\psi_{\alpha}\right\rangle=\alpha\left|\psi_{\alpha}\right\rangle
$$

which

$$
\left|\psi_{\alpha}\right\rangle=N_{0} \exp \left(-\frac{\Omega}{\hbar} e^{\gamma t} x^{2}-\alpha e^{-\gamma t / 2} x\right)
$$

The probability distribution for the dissipative system is

$$
\begin{aligned}
P(t) & =\int_{-\infty}^{\infty}\left|\psi^{*}(\varphi)\right\rangle\langle\psi(\varphi)| d \varphi \\
& =\int_{-\infty}^{\infty}\left|\psi^{*}(\psi)\right\rangle\langle\psi(\psi)| d \psi
\end{aligned}
$$

Therefore, the probability density is conserved in both Hilbert spaces.

\section{Conclusion}

In this paper, we consider the dissipative scalar field with time dependent frictional coefficient. We present a time dependent lagrangian similar to Bateman lagrangian for the dissipative massive scalar field. The properties of a quantum dissipative scalar field are analyzed by Semi-Caldirola-Kanai model. It is shown the probability density is conserved in both Hilbert spaces.

\section{REFERENCES}

[1] W. Paul, "Electromagnetic traps for charged and neutral particles," Rev. Mod. Phys. Vol. 62, pp. 531-540, 1990.

[2] D. Leibfried, R. Blatt, C. Monroe, and D. Wineland, "Quantum dynamics of single trapped ions," Rev. Mod. Phys. Vol. 75, pp. 281-324, 2003.

[3] E. Torrontegui, X. Chen, A. Ruschhaupt, and J.G. Muga, "Lewis-Riesenfeld invariants and transitionless quantum driving," Phys. Rev. A, Vol. 83, pp. 062116 (1-8), 2011.

[4] H. Johnston and S. Sarkar, "A re-examination of the quantum theory of optical cavities with moving mirrors," J. Phys. A, Vol. 29, pp. 1741-1746, 1996.

[5] M.S. Sarandy, E.I. Duzzioni, and R.M. Serra, "Quantum computation in continuous time using dynamic invariants," Phys. Lett. A, Vol. 375, pp. 3343 (1-7), 2011.

[6] U. Gungordu, Y. Wan, M.A. Fasihi, and M. Nakahara, "Dynamical invariants for quantum control of four-level systems," Phys. Rev. A, Vol. 86, pp. 062312 (1-9), 2012.

[7] M.C. Bertin, J.R.B. Peleteiro, and B.M. Pimentel, "Dynamical Invariants and Quantization of the One-Dimensional TimeDependent, Damped, and Driven Harmonic Oscillator,” Braz J Phys. Vol. 50, pp.534-540, 2020.

[8] L. Astrakhantsev and D. Oleksandr, "Massive scalar field theory in the presence of moving mirrors," Mod. Phys. Lett. A, Vol. 33, pp. 1850126 (1-24), 2018.

[9] L.H. Yu and C.P. Chang, "Evolution of the wave function in a dissipative system," Phys. Rev. A, Vol. 49, pp. 592-595, 1994. 
[10] L.H. Yu and C.P. Sun, "Quantum tunneling in a dissipative system," Phys. Rev. A, Vol. 54, pp. 3779 (1-13), 1996.

[11]H. Dekker, "classical and quantum mechanics of the damped harmonic oscillator," Phys. Rep, Vol.80, pp.1-110, 1981.

[12]C.I. Um, K.H. Yeon, and T.F. George, "The quantum damped harmonic oscillator," Phys. Rep. Vol. 362, pp. 63-192, 2002.

[13]H. Bateman, "on dissipative systems and related variational principles," Phys. Rev. Vol. 38, pp. 815-819, 1931.

[14]M.C. Huang and M.C. Wu, "The CaldirolaKanai model and its equivalent theories for a damped oscillator," Chin. J. Phys. Vol. 36, pp. 566-587, 1998.

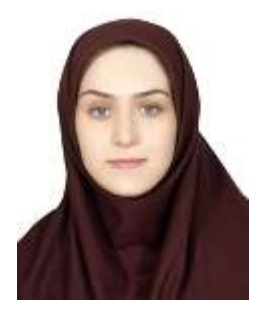

Marjan Jafari was born in Khorramdareh, Zanjan, on September 11, 1984. She received her B.Sc. in Solid State Physics in 2006. She also received her M.Sc. and Ph.D. degrees in Theoretical Physics from Physics Department of Isfahan University, Isfahan, Iran, under supervision of Professor Kheirandish, in 2009 and 2013, respectively. Dr Jafari research interests are Quantum Information, Quantum Optics, Quantum Field Theory, Casimir Effect, and Nanoelectromechanics. 
THIS PAGE IS INTENTIONALLY LEFT BLANK. 